\title{
A PROSPECTIVE STUDY OF ADVERSE DRUG REACTIONS IN A TERTIARY CARE HOSPITAL IN SOUTH INDIA
}

\author{
JERIN JAMES*, JAMUNA RANI \\ Department of Pharmacology, SRM Medical College, Chennai, Tamil Nadu, India. Email: jerinjames06@gmail.com
}

Received: 10 October 2019, Revised and Accepted: 15 November 2019

\begin{abstract}
Objective: The objective of this study was to determine the prevalence of adverse drug reactions (ADRs) in a tertiary care teaching hospital in South India and to analyze the causality according to the Naranjo scale.

Methods: This study was carried out at the SRM Medical College, Chennai, a tertiary care hospital, which is an ADR monitoring center under Pharmacovigilance Programme of India, over a period of 1 year, from September 2017 to August 2018, after approval by the Institutional Ethics Committee. These ADRs were reported by the clinical pharmacy team of our hospital to the department of pharmacology. The relevant data were collected from the patient case notes, treatment charts, laboratory data reports, ADR notification forms, patient interview and reporter interviews after written informed consent was obtained from each patient. Patients of either sex of all ages who developed ADR within the hospital were included in the study. Patients who developed ADR outside the hospital were excluded from the study. The collected ADRs were subjected to the Naranjo causality assessment scale. Hartwig's scale was used to assess the severity of the reaction. The causalities and other aspects of the ADRs were analyzed in detail.
\end{abstract}

Results: A total of 80 ADRs were reported during this study period. The ADRs were most frequently reported in the adult age group (75\%) with slight female preponderance (60\%). Antibiotics contributed to the maximum number of ADRs which accounted for $52.5 \%$ of the total, of which betalactams were the highest (37.5\%) followed by analgesics. Causality assessment of ADRs by the Naranjo scale showed that the maximum reactions could be categorized as probable (85\%) followed by the possible category (15\%). The most common presentation of ADR was itching (45\%) followed by rashes (30\%). Three cases of severe adverse reactions were reported, one case each of anaphylaxis to pantoprazole and diclofenac and one case of Steven-Johnson syndrome to cotrimoxazole.

Conclusion: ADRs are a common occurrence but are often not recognized. Even if recognized, they are underreported as many physicians are unaware that all ADRs should be reported to ADR monitoring centers. ADRs are an important cause of morbidity and mortality all over the world and are an important public health concern. It inflicts a negative impact on the treatment and exerts a greater economic burden on the patient if it results in prolongation of the duration of hospitalization or other comorbidities. Therefore, the practicing physicians, as well as the nursing staff, should be sensitized of the importance of ADR reporting to their respective pharmacovigilance centers.

Keywords: Adverse drug reactions, Pharmacovigilance, Naranjo scale.

(C) 2020 The Authors. Published by Innovare Academic Sciences Pvt Ltd. This is an open access article under the CC BY license (http://creativecommons. org/licenses/by/4. 0/) DOI: http://dx.doi.org/10.22159/ajpcr.2020.v13i1.36028

\section{INTRODUCTION}

Adverse drug reactions (ADRs) are as old as medicines. The World Health Organization defines an ADR as "any response to a drug which is noxious and unintended, and which occurs at doses normally used in man for prophylaxis, diagnosis, or therapy of disease, or the modification of physiological function [1]." In other words, an ADR is harm directly caused by the medicine at normal doses, during normal use. The impact of morbidity and mortality associated with ADRs poses a greater economic burden on the health-care system, especially in a developing country like India [2]. However, the incidence of spontaneous reporting is on the lower side due to the lack of awareness of the need for reporting [3-5]. In this context, the importance of pharmacovigilance arises. Pharmacovigilance helps in the early detection of ADRs, identification of risk factors, and understanding the mechanisms underlying the ADR [6].

Pharmacovigilance is defined as "the science and activities relating to the detection, assessment, understanding, and prevention of adverse effects or any other possible drug-related problems [6]." The Central Drugs Standard Control Organization and the Directorate General of Health Services under the Ministry of Health and Family Welfare, Government of India, in collaboration with the Indian Pharmacopoeia Commission, Ghaziabad, conduct nation-wide Pharmacovigilance Program of India
(PvPI) to protect the health of the patient by assuring drug safety. The PvPI aims to safeguard the health of the Indian population by ensuring that the benefits of the use of medicine outweigh the risks associated with its use [7]. The vision of PvPI is to improve patient safety and thereby reducing the risk associated with the use of medicines.

The SRM ADR monitoring center is designated under PvPI and is working for the safety and welfare of patients, in coordination with all the clinical and respective paramedical departments by prompt detection, reporting, and monitoring of the ADR and providing appropriate management. As ADRs are an inevitable part of treatment, it is essential to diagnose those and sensitizes physicians to report those as it will help the budding physicians in the right management of cases. Hence, this study aims to analyze the ADRs reported from our hospital so that physicians can anticipate the following ADRs while prescribing these classes of drugs and be cautious while prescribing them in future.

\section{METHODS}

The study was designed as a prospective spontaneous reporting study involving active methods (pharmacist actively seeking suspected ADRs) and passive methods (stimulating clinicians to report suspected ADRs) and was carried out in all departments of the hospital, for a period of 1 year. Eighty patients of all age groups who developed ADRs 
were included for the study. Written informed consent was obtained from the patients. The data for the study were taken from case sheets, investigation reports, personal interviews with clinicians and personal interviews with the patient or patient's attendant, history of medications, and reports of medical and surgical interventions.

The causality assessment of the reported ADRs was done using the Naranjo causality assessment scale into definite, probable, or possible or doubtful [8]. The modified Hartwig's and Siegel scale defines the severity of ADR as mild, moderate, or severe.

\section{RESULTS}

A total number of 80 ADRs were reported during the 12 months of study duration from September 2017 to August 2018, of which 60\% were female and $40 \%$ were male (Fig. 1). Pediatric patients experienced ( $<18$ years) $2.5 \%$ ADR followed by the geriatric age group ( $>60$ years) $22.5 \%$ and those in the adult age group between 18 and 59 years are $75 \%$ ADRs (Fig. 2).

The highest number of ADRs was reported from general medicine (25\%) followed by the general surgery department (17.5\%) (Fig. 3).

The majority of ADRs were due to antimicrobial agents (52.5\%), of which beta-lactams were the highest (37.5\%) followed by ciprofloxacin (15\%) (Fig. 4).

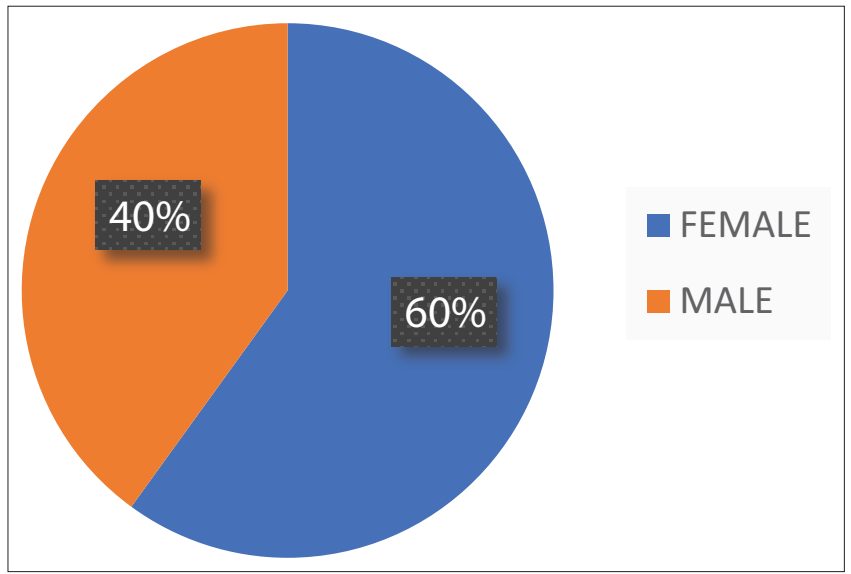

Total number of cases $-\mathbf{8 0}$

Fig. 1: Gender distribution in reported adverse drug reactions

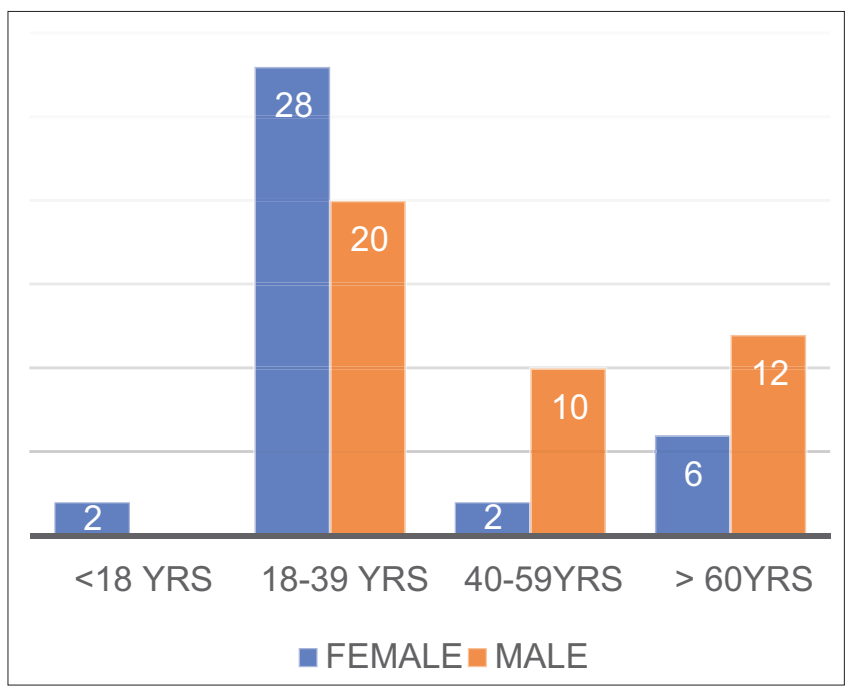

Total number of cases - 80

Fig. 2: Age distribution in reported adverse drug reactions
The presentations of ADR were manifold, itching (36 cases) was the most common presentation reported followed by rashes (24) and shivering (10) (Table 1).

The most commonly affected organ system was found to be the skin $(75 \%)$ followed by the central nervous system $(12.5 \%)$ and gastrointestinal systems (7.5\%) (Fig. 5).

Most of the reactions were mild (75\%) as per modified Hartwig's criteria (Fig. 6).

Causality assessment was done using the Naranjo scale which showed that $85 \%$ of the reactions were probable and $15 \%$ were possible (Fig. 7 ).

\section{DISCUSSION}

The majority of ADRs (75\%) were seen in younger adults in the age group of 18-39 years. This was comparable with the previous study by Kumar et al. in Himachal Pradesh, India [9]. This could be due to a greater number of patients getting admitted for treatment in this age group. Furthermore, female preponderance was noted among the cases (60\%). Peter et al., in his study conducted in Tamil Nadu, had also observed a greater incidence of ADR among females [10]. The most frequent ADRs were due to antibiotics which could be related to increased frequency of prescription of antibiotics. Similar results were obtained by Raut et al. in their study conducted in Maharashtra where the maximum number of ADRs was found to be associated with antibiotics [11]. Among the ADRs,

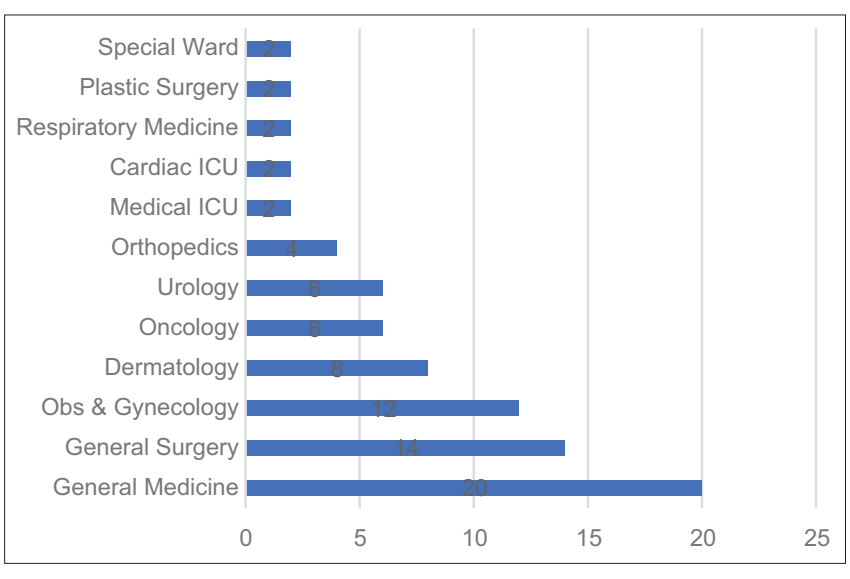

Total number of cases $-\mathbf{8 0}$

Fig. 3: Adverse drug reaction reported from various departments

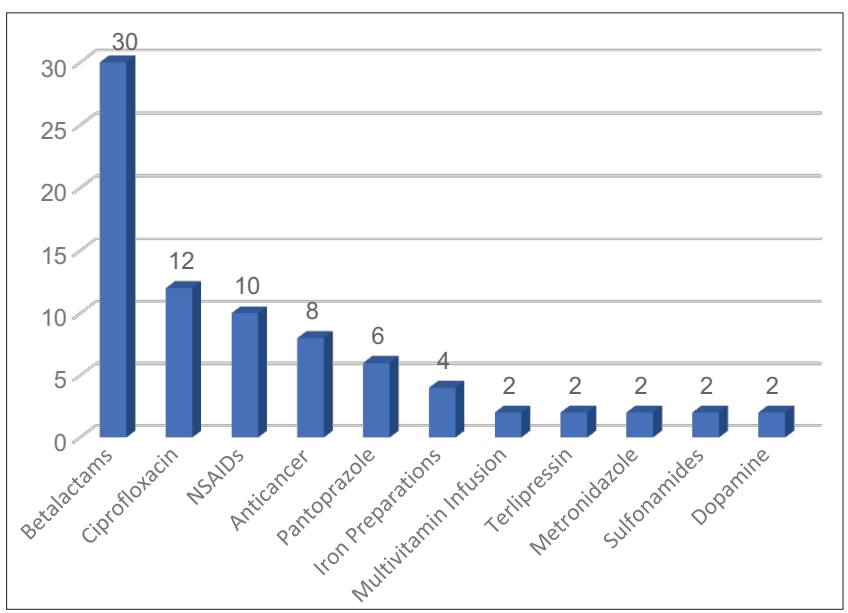

Total number of cases - 80

Fig. 4: Drugs in reported adverse drug reactions 
Table 1: List of presentations of ADRs

\begin{tabular}{|c|c|c|}
\hline Reaction & Drugs & Number of ADRs \\
\hline Itching & $\begin{array}{l}\text { Piperacillin with tazobactam, ciprofloxacin, penicillin, amoxicillin with clavulanate, NSAIDs, } \\
\text { ceftriaxone with tazobactam cefuroxime, cefoperazone, and dopamine }\end{array}$ & 36 \\
\hline Rashes & $\begin{array}{l}\text { Methotrexate, cefoperazone+sulbactam, cefixime, amoxicillin with clavulanate, tramadol, cefotaxime, } \\
\text { and paclitaxel }\end{array}$ & 24 \\
\hline Shivering & Iron+folic acid, Iron & 10 \\
\hline Vomiting & Ceftriaxone, oxaliplatin & 3 \\
\hline Mouth ulcers & Sulfa drug, metronidazole & 2 \\
\hline Breathing difficulty & Pantoprazole, diclofenac & 2 \\
\hline Diarrhea & Terlipressin & 1 \\
\hline
\end{tabular}

Total number of cases - 80. ADR: Adverse drug reactions, NSAIDs: Nonsteroidal anti-inflammatory drugs

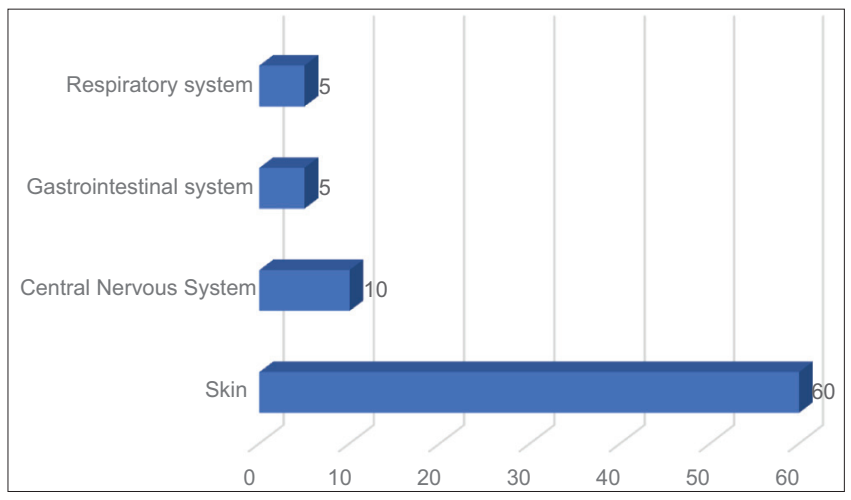

Total number of cases $-\mathbf{8 0}$

Fig. 5: Organ system affected due to adverse drug reactions

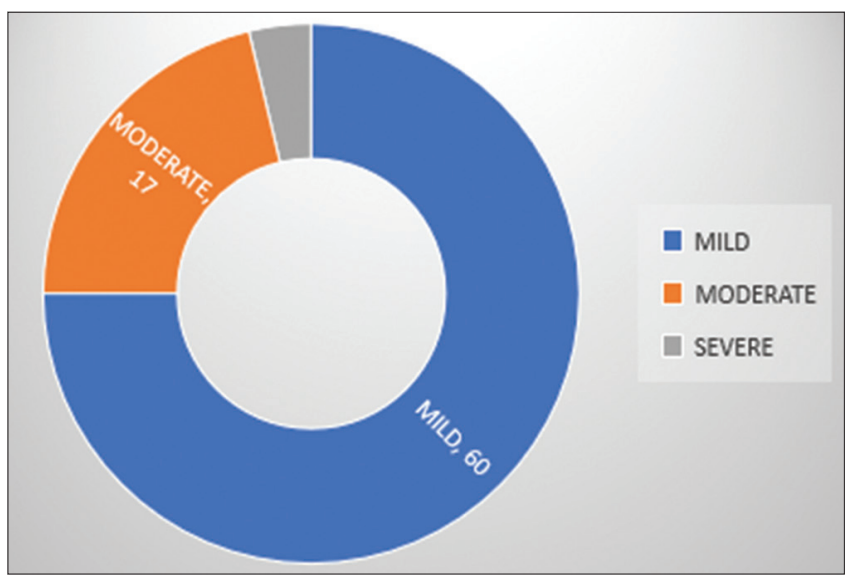

Total number of cases -80

Fig. 6: Severity of reported adverse drug reactions

major proportions of adverse reactions were seen with beta-lactam antibiotics $(37.5 \%)$ which were similar to the observations by Akalu and Belavadi as well as by Jung et al. $[12,13]$. Beta-lactam antibiotics are one of the widely used antibiotics for a wide variety of infections. The number of ADRs was highest in general medicine followed by general surgery departments. This is due to the wide use of antibiotics in these departments for the treatment and prophylaxis of various infections. Also, the patients admitted in these departments have comorbidities that require polypharmacy, which accounts for the high number of ADRs. Following the previous studies by Mahatme and Narasimharao, Gupta et al., and Jung et al., the present study showed the predominance of cutaneous manifestations on presentation of ADRs (75\%) [13-15] followed by central nervous system manifestations such as dizziness. However, several other studies have observed gastrointestinal symptoms such as vomiting and diarrhea as the most common manifestation of

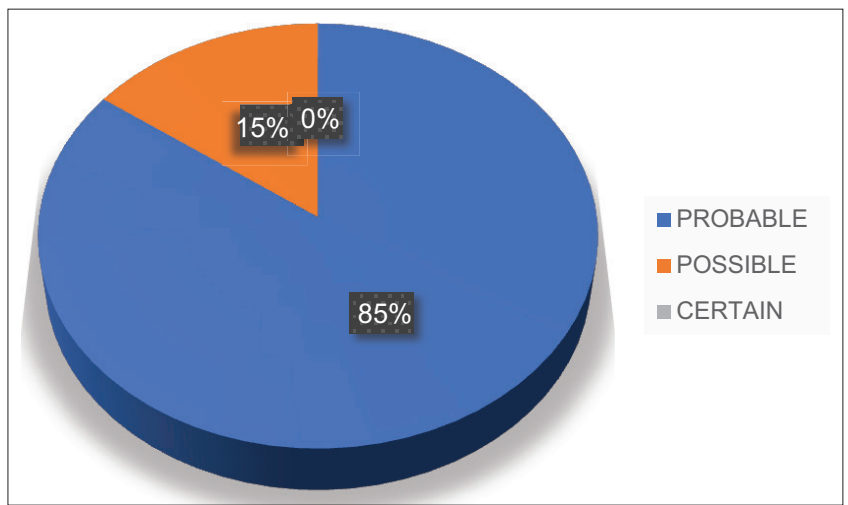

Total number of cases -80

Fig. 7: Causality assessment using the Naranjo scale

ADR [16-18]. The drug dechallenge was done in most of the cases and in some, the dose was reduced while no changes were made in others considering the risk-benefit ratio in particular patients. The majority of the patients recovered completely from the effects of ADR within a day since most of the reactions were mild (60\%) according to the modified Hartwig's criteria. About $17 \%$ of the cases were moderate ADRs requiring interventions, while there were three cases that there was life-threatening requiring artificial ventilation and intensive care unit support. The causality assessment of the reported ADRs according to the Naranjo scale revealed that no reactions were certain and most of them were probably with a lesser number of possible ADRs. No reactions were unlikely. Hence, from the above discussion, we can conclude that there is a similar pattern in the occurrence of ADRs from the drugs implicated to the manifestations that occur.

\section{CONCLUSION}

There is an increasing need for interventions for the prevention of ADR-related health problems. Better knowledge of preventable ADRs could help to design strategies to protect patients from being affected by ADRs. The rational use of medicines by prescribing lower doses and prefer not to use multiple therapies in the very first stage of medication should be encouraged. Health professionals such as nurses or pharmacists play a major role in monitoring drug therapy prescribed by the medical practitioners as they provide information to patients about medications and their rational use and also monitor the health and progress of patients in response to medications to ensure its safety and efficacy. Hence, health professionals should periodically be educated about adverse reactions and should be encouraged for spontaneous reporting. Pharmaceutical industries have also started taking part in pharmacovigilance program and have taken initiative by setting up a separate cell and self-online reporting system to counteract any adverse effects of their products. Even the safety information of pharmaceutical products is communicated to doctors by pharmaceutical companies in the form of "dear doctor letters [19]." This study aims to emphasize the awareness of the health-care providers on 
vigilant monitoring of ADRs and to encourage prompt reporting of the same to prevent the occurrence of ADRs. The present study had some limitations as it is an observational study of short duration; still, this would give an insight into the current situation and of trends in ADRs in tertiary health-care centers and will help to increase awareness for further pharmacovigilance activities.

\section{ACKNOWLEDGMENT}

I am grateful to our respectable Medical Director, Dean (Medical), Medical Superintendent for their support and granting permission to carry out this study. I also thank my teachers and my colleagues for helping me in this study.

\section{AUTHORS' CONTRIBUTIONS}

I, Jerin James, was involved in the entire project from designing protocol for the study, data collection, statistical analysis, and manuscript writing. The coauthor, Dr. R. Jamuna Rani, was involved throughout in providing the right guidance, choosing the research topic, and rectifying clarifications whenever required.

\section{CONFLICTS OF INTEREST}

Declared none.

\section{REFERENCES}

1. Tripathi KD. Essentials of Medical Pharmacology. $7^{\text {th }}$ ed. New Delhi: JP Brothers; 2018. p82-91.

2. Daulat MP, Ambika VJ, Singh P, Raj B. A prospective study of adverse drug reactions in a tertiary care teaching hospital. Int J Basic Clin Pharmacol 2018;7:1965-9.

3. Kaufman G. Adverse drug reactions: Classification, susceptibility, and reporting. Nurs Stand 2016;30:53-63.

4. Mulchandani R, Kakkar AK. Reporting of adverse drug reactions in India: A review of the current scenario, obstacles, and possible solutions. Int J Risk Saf Med 2019;30:33-44.

5. Laila KV, Hemalatha $\mathrm{T}$. Awareness of adverse drug reactions reporting among doctors in a tertiary care center. Int $\mathrm{J}$ Basic Clin Pharmacol 2016;5:2236-9.
6. Suke SG, Kosta P, Negi H. Role of pharmacovigilance in India: An overview. Online J Public Health Inform 2015;7:e223.

7. Kalaiselvan V, Srivastava S, Singh A, Gupta SK. Pharmacovigilance in India: Present scenario and future challenges. Drug Saf 2019;42:339-46.

8. Zaki SA. Adverse drug reaction and causality assessment scales. Lung India. 2011;28:152-3.

9. Kumar A, Kansal D, Sharma P, Bhardwaj A, Sawaraj S. To study the pattern of adverse drug reactions among patients hospitalized in the medical wards of a tertiary care hospital. Int J Basic Clin Pharmacol 2016;5:1972-7.

10. Peter JV, Varghese GH, Alexander H, Tom NR, Swethalekshmi V, Truman C, et al. Patterns of adverse drug reaction in the medical wards of a teaching hospital: A prospective observational cohort study. Curr Drug Saf 2016;11:164-71.

11. Raut A, Pawar A, Pankaj M, Srivastava P, Mishra A. Clinical pattern and severity of cutaneous adverse drug reactions. Int J Pharm Pharm Sci 2013;5:612-6.

12. Akalu SD, Belavadi NG. Pattern of adverse drug reactions due to antibiotics in a tertiary care hospital. Int $\mathrm{J}$ Basic Clin Pharmacol 2017;6:169-80.

13. Jung IY, Kim JJ, Lee SJ, Kim J, Seong H, Jeong W, et al. Antibioticrelated adverse drug reactions at a tertiary care hospital in South Korea. Biomed Res Int 2017;2017:4304973.

14. Mahatme N, Narasimharao R. A study of clinical patterns and causative agents of adverse cutaneous drug reactions. Indian J Drugs Dermatol 2016;2:13-5

15. Gupta A, Kaur A, Shukla P, Chhabra H. Adverse drug reactions pattern in a tertiary level teaching hospital: A retrospective study. Indian J Pharm Pract 2017;10:27-31.

16. Khan F, Nizamuddin S, Huda N, Mishra H. A prospective study on prevalence of adverse drug reactions due to antibiotics usage in otolaryngology department of a tertiary care hospital in North India. Int J Basic Clin Pharmacol 2013;2:548-53.

17. Alsbou M, Alzubiedi S, Alzobi H, Samhadanah NA, Alsaraireh Y, Alrawashdeh $\mathrm{O}$, et al. Adverse drug reactions experience in a teaching hospital in Jordan. Int J Clin Pharm 2015;37:1188-93.

18. Velraj M. A prospective observational study on pattern of adverse drug reaction to antibiotics commonly prescribed in the hospitalized pediatric patients. Asian J Pharm Clin Res 2018;11:118-20.

19. Kumar L. Pharmacovigilance/reporting adverse drug reactions: An approach to enhance health surveillance and extending market share by minimizing the chances of drug withdrawals. Int J Pharm Pharm Sci 2015;7:1-7. 